\title{
Dynamics of optically switched bistable laser diodes in the injection-locked state
}

\author{
R. Hui, A. Paradisi, S. Benedetto, and I. Montrosset \\ Dipartimento di Elettronica, Politecnico di Torino, Corso Duca degli Abruzzi 24, 10129 Torino, Italy
}

Received May 19, 1993

\begin{abstract}
The dynamical properties of dispersive optical bistability in a semiconductor laser biased from below to above threshold are investigated both experimentally and theoretically. The optical bistability switch-off time is found to decrease continuously from below to above threshold. A fast switch-off in less than $100 \mathrm{ps}$ has been observed when the laser operates in the injection-locked condition.
\end{abstract}

Bistable laser diodes are expected to be key components in future optical communication and switching systems because of their advantages such as inherent optical gain and low optical switching power. One of the most important remaining problems with such devices is their limited switching speed. When a semiconductor laser, biased just below threshold, was operated as an optical amplifier, dispersive optical bistability (OB) has been demonstrated, ${ }^{1-4}$ and the OB switch-off time is usually considered to be limited by the inverse of the effective carrier lifetime. On the other hand, OB has also been found recently in optically injection-locked semiconductor lasers. Injection locking can be achieved either by sweeping the injected optical power with a fixed frequency detuning near the stable locking band or by sweeping the frequency detuning with the fixed optical power. 5,6 The hysteresis width in the latter case was found to be determined by the ratio between the input optical power and the optical power emitted by the slave laser. $^{6}$ It should be pointed out, however, that since the laser diode now works above threshold, in the off state of $\mathrm{OB}$, optical injection locking is no longer maintained, and the free-running slave mode results. In practical applications only the optical power in the locked frequency is required, and the unwanted freerunning slave mode has to be removed; therefore an optical filter is necessary.

In this Letter we present our experimental measurements of the dynamical properties of $O B$ in a distributed-feedback semiconductor laser biased from below to above threshold. Since the switch-on time usually depends on the signal optical power, the switch-off time is more important because it depends mainly on the device characteristic. The limit to the OB switch-off time can be evaluated through the dynamics of the junction electric voltage because this voltage signal is proportional to the variation of the carrier density inside the laser cavity and the carrier recovery is slower than the optical switch-off. ${ }^{7}$

In addition to the previous observation that the switch-off time becomes smaller as the injection current is increased toward the threshold level, ${ }^{8}$ our measured result revealed that the switch-off can even be much faster when the slave laser is biased far above threshold. The results of computer simulation with the rate-equation model qualitatively agree with that of the experiment.

The experimental setup shown in Fig. 1 is similar to that described in Ref. 7, except for the modulation of the signal laser and the presence of a third laser diode (LD3), the master laser used for optical injection locking LD1 to prevent frequency chirp when LD1 is directly modulated.

LD1 is directly current modulated by a sinusoidal wave at $300 \mathrm{MHz}$ by a bias tee. LD2 is then optically switched by the light injected from LD1. Since the carrier recovery is the main limitation to the $O B$ switch-off time, we measured the carrier population variation through the laser junction electric voltage signal. This measurement is based on the fact that the junction electric voltage signal is proportional to the variation of carrier density inside the laser cavity through the change in the quasi-Fermi level. Figure 2(a) shows the junction electric voltage waveform during switch-off when LD2 is biased at $99 \%$ of the threshold; the switch-off time is $\sim 1$ ns. If LD2 is biased above threshold, it operates in the optically injection-locking regime, and bistability occurs near the edge of the stable locking band. ${ }^{6}$ In this case, however, the $\mathrm{OB}$ switch-off time is found to be much smaller than in the previous case; the waveform during switch-off is shown in Fig. 2(b), where LD2 is biased at 2.75 times the threshold. An OB switch-off

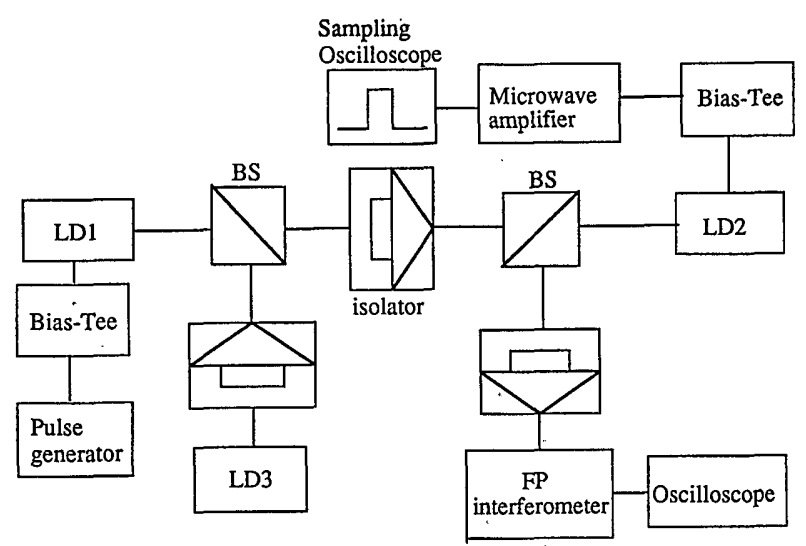

Fig. 1. Experimental setup. LD's, laser diodes; BS's, beam splitters; FP, Fabry-Perot. 
(a)

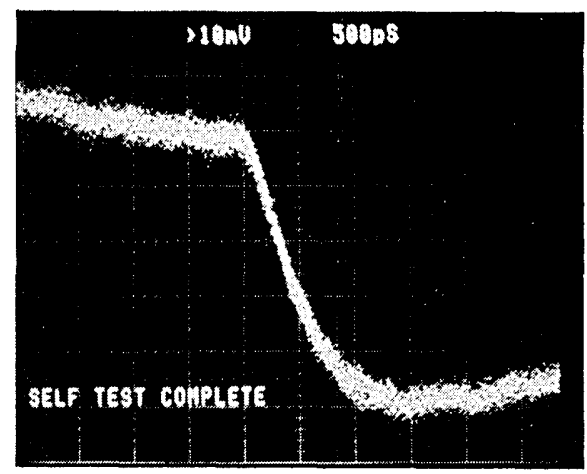

(b)

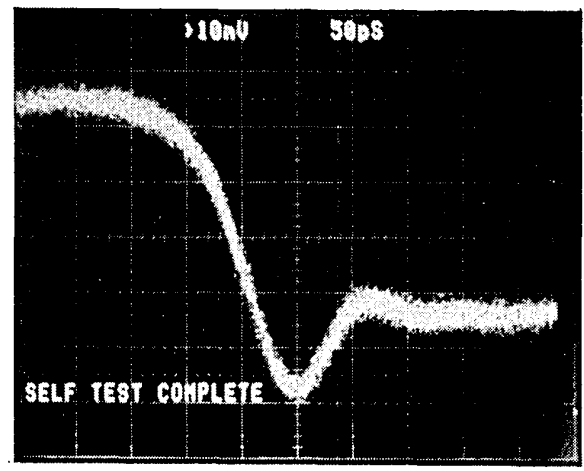

Fig. 2. Measured OB switch-off waveform with LD2 biased at (a) 0.99 and (b) 2.75 times the threshold current.

time of less than 100 ps is shown in Fig. 2(b), which is much shorter than the effective carrier lifetime $(1.4 \mathrm{~ns})$ of this laser. A systematic measurement of the OB switch-off time versus the injection current of LD2 from below to above threshold has been also performed, and the result is given in Fig. 3 (circles); the switch-off time keeps decreasing with increasing bias level. For all the measurements, the wavelengths of the signal laser were chosen at the center of the bistable loop at the steady state.

Our theoretical analysis is based on the rateequation model $^{9}$ :

$$
\frac{\mathrm{d} E}{\mathrm{~d} t}=\frac{1}{2}\left[\Gamma G(N, P)-1 / \tau_{p}\right] E+i \Delta \Omega E+\frac{1}{\tau_{i}} E_{\text {ext }},
$$

$$
\begin{aligned}
& \frac{\mathrm{d} P_{s}}{\mathrm{~d} t}=\left[\Gamma G(N, P)-1 / \tau_{p}\right] P_{s}+R_{\mathrm{sp}}, \\
& \frac{\mathrm{d} N}{\mathrm{~d} t}=I / q-R(N)-G(N, P) P .
\end{aligned}
$$

where $P=P_{s}+|E|^{2}$ is the total photon number in the slave laser's active cavity, $E$ is the normalized electric field generated in response to the externally injected optical source $E_{\text {ext }}, P_{s}$ is the photon number generated in response to the spontaneous emission in Eq. (2), $\Delta \Omega=\Omega-\omega$ is the relative detuning between the cavity resonance frequency of the master and the slave lasers, and $G(N, P)=$ $G_{N}\left(N-N_{0}\right)-G_{I} P$ is the material gain, with $G_{N}$ the differential gain, $G_{I}$ the nonlinear gain, $N$ the carrier number, and $N_{0}$ the carrier number at transparency. $\tau_{p}$ is the photon lifetime, $\tau_{i}$ is the cavity round-trip time, $\Gamma$ is the confinement factor,
$I$ is the electrical current, $q$ is the electron charge, and the carrier dependence of the refractive index is represented by $\alpha=-2(\partial \omega / \partial N) / G_{N} . \quad R(N)=A N+$ $B N^{2}+C N^{3}$ represents the carrier recombination effect, with $A, B$, and $C$ the nonradiative, radiative, and Auger recombination coefficients, respectively. The spontaneous emission rate in Eq. (2) can therefore be assumed to be $R_{\mathrm{sp}}=\beta_{\mathrm{sp}} B N^{2}$, where $\beta_{\mathrm{sp}}$ is the spontaneous emission coefficient that represents the fraction of spontaneous emission coupled into the lasing mode.

The rate equations (1)-(3) have been solved numerically in the time domain with a fourthorder Runge-Kutta method. In order to extract the switch-off time easily, we chose a square wave as the optical input; in Figs. 4 and 5 the input optical signal switches on and off at 12 and $16 \mathrm{~ns}$, respectively, from 0 to $0.316 \mathrm{~mW}(-5 \mathrm{dBm})$. The frequency detuning was chosen such that, at 0.158-mW constant optical injection, the signal wavelength was at the center of the frequency bistable loop. The calculated carrier population variation in the time domain is given in Fig. 4, with the laser biased at $I=0.95 I_{\text {th }}$ (dashed curve) and $I=2.7 I_{\text {th }}$ (solid curve). Obviously, switch-off

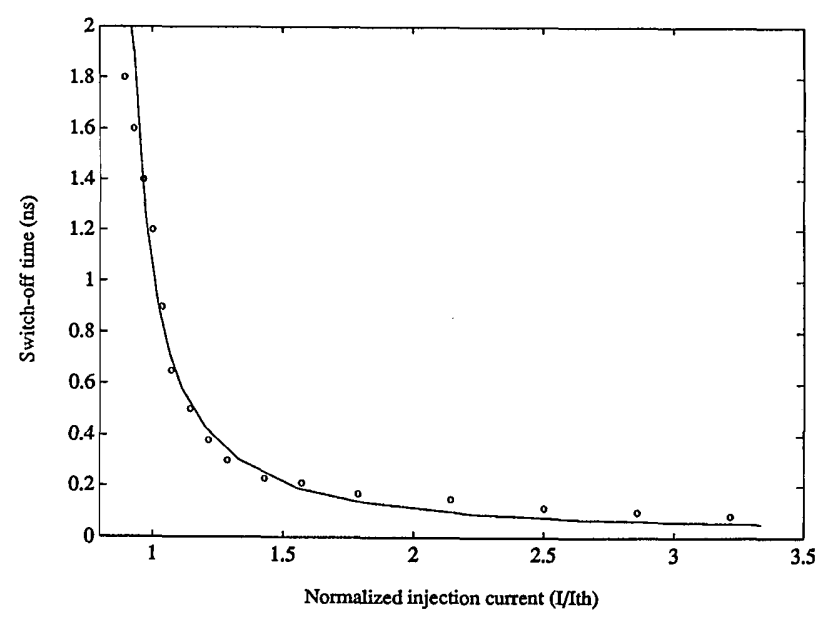

Fig. 3. Measured (circles) and calculated (sold curve) OB switch-off times.

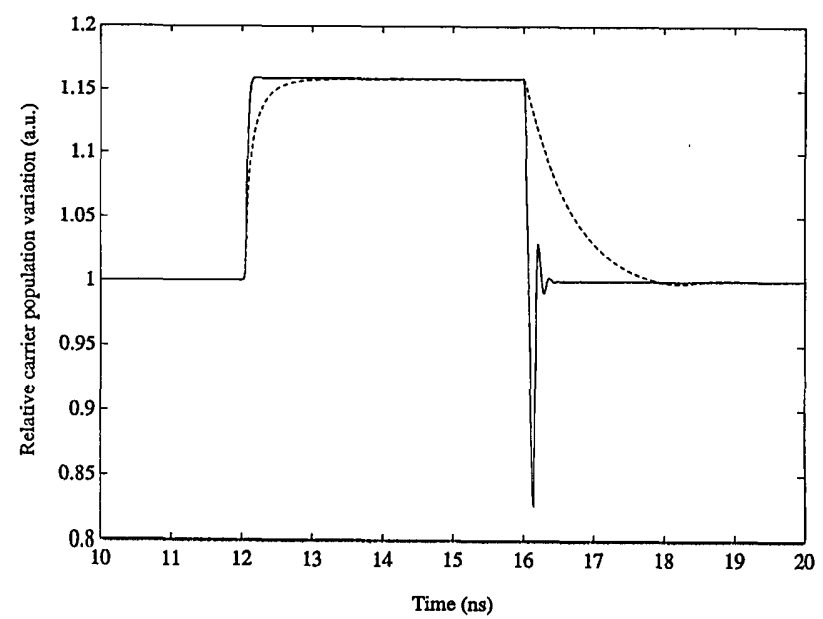

Fig. 4. Calculated relative carrier population variation with the laser diode biased at $I=0.95 I_{\text {th }}$ (dashed curve) and $I=2.7 I_{\text {th }}$ (solid curve). 


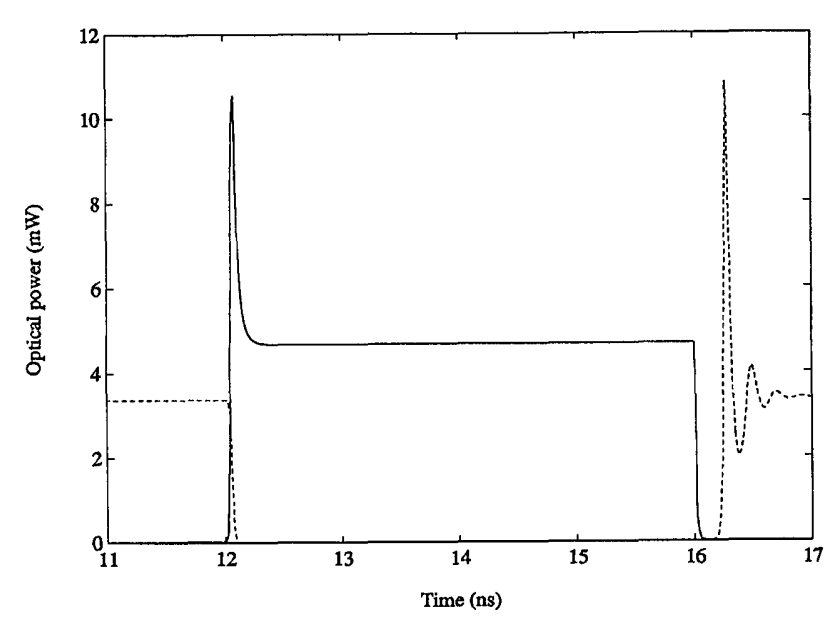

Fig. 5. Output optical power at the signal wavelength (solid curve) and the stimulated emission of the slave laser (dashed curve). The slave laser is biased at $I=2 I_{\text {th }}$.

is much faster in the latter case, and ripple at the switch-off edge is present, which reflects the effect of relaxation oscillation. Qualitative agreement between these calculated results and the measured results in Fig. 2 is obtained. However, the spike at switch-off is more pronounced in the calculated waveform than in the measured one when the laser operates far above threshold. This may be caused by the limited bandwidth $(12 \mathrm{GHz})$ of the microwave amplifier used in the experiment and the parasitic effect in the laser package.

The calculated OB switch-off time versus the normalized injection current is plotted in Fig. 3 as a solid curve, which agrees well with the experiment. $\mathrm{Pa}$ rameters used in the calculation are $\alpha=6, G_{N}=8 \times$ $10^{3} \mathrm{~s}^{-1}, G_{I}=6.8 \times 10^{5} \mathrm{~s}^{-1}, N_{0}=5 \times 10^{7}, \tau_{i}=5.5 \mathrm{ps}$, $\tau_{p}=1 \mathrm{ps}, A=10^{8} \mathrm{~s}^{-1}, B=3 \mathrm{~s}^{-1}, C=4 \times 10^{-9} \mathrm{~s}^{-1}$, $\beta_{\mathrm{sp}}=10^{-5}$, and $v=5 \times 10^{-11} \mathrm{~cm}^{3}$. These are typical values for bulk distributed-feedback semiconductor lasers. With these values, the spontaneous emission carrier lifetime at threshold is $1.4 \mathrm{~ns}$, which is equal to that experimentally measured in LD2.

The physical reason for this fast switching is that during the switch-off transient there is a competition between the amplified input optical signal and the stimulated emission of the slave laser, when it operates above threshold. This is demonstrated in Fig. 5 , with $I=2 I_{\text {th }}$. The carrier dynamics is no longer governed by the effective carrier lifetime, but rather it is determined by the inverse of the relaxation-oscillation frequency of the slave laser. Therefore much faster switch-off can be obtained if the slave laser is biased at relatively high levels. The limitation of the bias level is set by the increase of the optical signal required to be injected into the slave laser to achieve the bistable operation. ${ }^{6}$ From the application point of view, since the slave laser operates above threshold, an optical filter is usually required for removal of the stimulated emission from the slave laser itself. At switch-off, the frequency difference between the signal and the free-running slave mode can be of the order of tens of gigahertz, depending on the ratio between the injected optical power and the power of the slave laser. ${ }^{6}$ One can filter out this free-running slave mode by using, for example, an integrated Mach-Zehnder interferometric optical filter. In order to filter out the stimulated emission easily from the slave laser, one can either increase the signal optical power or decrease the bias level of the slave laser. However, the former is limited by the power of the semiconductor laser sources available and the latter will result in an increase of the transient time. Therefore a tradeoff between the switching time and the possibility of filtering has to be considered in the practical application.

In conclusion, the $\mathrm{OB}$ switch-off time in an optically switched semiconductor laser is found to decrease continuously with the laser biased from below to above threshold. A fast OB switch-off, in less than $100 \mathrm{ps}$, has been observed experimentally when the laser operates far above threshold in the injectionlocked regime. To our knowledge, this is the fastest switch-off in dispersive $O B$ of semiconductor lasers ever reported. ${ }^{10}$

This work was supported by the Consiglio Nazionale Delle Ricerche and the Camera di Commercio di Torino under the research grant All-Optical Communication Networks and by the Ministero per Universita Ricerca Scientifica Tecnologica.

\section{References}

1. K. Otsuka and H. Iwamura, IEEE J. Quantum Electron. QE-19, 1184 (1983).

2. K. Otsuka and S. Kobayashi, Electron. Lett. 19, 262 (1983).

3. A. J. Adams, H. J. Westlake, M. J. Mahony, and I. D. Henning, IEEE J. Quantum Electron. QE-21, 1498 (1985).

4. M. J. Adams, Proc. Inst. Electr. Eng. Part J 132, 343 (1985).

5. H. Kawaguchi, K. Inoue, T. Matsuoka, and K. Otsuka, IEEE J. Quantum Electron. QE-21, 1314 (1985).

6. R. Hui, A. D'Ottavi, A. Mecozzi, and P. Spano, IEEE J. Quantum Electron. 27, 1688 (1991).

7. R. Hui, S. Benedetto, and I. Montrosset, Opt. Lett. 18, 287 (1993).

8. W. F. Sharfin and M. Dagenais, IEEE J. Quantum Electron. QE-23, 303 (1987).

9. R. Lang, IEEE J. Quantum Electron. QE-18, 976 (1982).

10. H. Kawaguchi, Proc. Inst. Electr. Eng. Part J 140, 3 (1993). 